\title{
BMJ Open Implementation of a novel stratified PAthway of CarE for common musculoskeletal (MSK) conditions in primary care: protocol for a multicentre pragmatic randomised controlled trial (the PACE MSK trial)
}

\author{
Trudy Rebbeck (D) , ${ }^{1,2}$ Kerrie Evans (D) , ${ }^{1,3}$ Paulo Ferreira, ${ }^{1}$ Darren Beales (D) , \\ Michele Sterling, ${ }^{5,6} \mathrm{Kim}$ L Bennell, ${ }^{7}$ Ian Cameron, ${ }^{1}$ Michael Nicholas, ${ }^{8}$ \\ Carrie Ritchie (D) , ${ }^{5,6}$ Gwen Jull, ${ }^{9}$ Julia Treleaven, ${ }^{9}$ Lyndal Trevena (D) , \\ Kathryn Refshauge, ${ }^{1}$ Luke Connelly, ${ }^{10}$ Nadine Foster, ${ }^{11,12}$ Deborah Black (D) , \\ Paul Hodges, ${ }^{9}$ Manuela Ferreira, ${ }^{1,8}$ Tim J Shaw, ${ }^{1}$ Milena Simic ${ }^{1}$
}

To cite: Rebbeck T, Evans K, Ferreira P, et al. Implementation of a novel stratified PAthway of CarE for common musculoskeletal (MSK) conditions in primary care: protocol for a multicentre pragmatic randomised controlled trial (the PACE MSK trial). BMJ Open 2021;11:e057705. doi:10.1136/ bmjopen-2021-057705

- Prepublication history and additional supplemental material for this paper are available online. To view these files, please visit the journal online (http://dx.doi.org/10.1136/ bmjopen-2021-057705).

Received 18 0ctober 2021 Accepted 23 November 2021

Check for updates

(C) Author(s) (or their employer(s)) 2021. Re-use permitted under CC BY-NC. No commercial re-use. See rights and permissions. Published by BMJ.

For numbered affiliations see end of article.

Correspondence to

Dr Kerrie Evans;

kerrie.evans@sydney.edu.au

\section{ABSTRACT}

Introduction Musculoskeletal (MSK) conditions constitute the highest burden of disease globally, with healthcare services often utilised inappropriately and overburdened. The aim of this trial is to evaluate the effectiveness of a novel clinical PAthway of CarE programme (PACE programme), where care is provided based on people's risk of poor outcome.

Methods and analysis Multicentre randomised controlled trial. 716 people with MSK conditions (low back pain, neck pain or knee osteoarthritis) will be recruited in primary care. They will be stratified for risk of a poor outcome (low risk/high risk) using the Short Form Örebro Musculoskeletal Pain Screening Questionnaire (SFÖMSPQ) then randomised to usual care $(n=358)$ or the PACE programme $(n=358)$. Participants at low risk in the PACE programme will receive up to 3 sessions of guideline based care from their primary healthcare professional (HCP) supported by a custom designed website ( mypainhub.com). Those at high risk will be referred to an allied health MSK specialist who will conduct a comprehensive patient-centred assessment then liaise with the primary HCP to determine further care. Primary outcome (SF 12-item PCS) and secondary outcomes (eg, pain self-efficacy, psychological health) will be collected at baseline, 3, 6 and 12 months. Cost-effectiveness will be measured as cost per quality-adjusted life-year gained. Health economic analysis will include direct and indirect costs. Analyses will be conducted on an intention-to-treat basis. Primary and secondary outcomes will be analysed independently, using generalised linear models. Qualitative and mixed-methods studies embedded within the trial will evaluate patient experience, health professional practice and interprofessional collaboration.

Ethics and dissemination Ethics approval has been received from the following Human Research Ethics Committees: The University of Sydney (2018/926), The University of Queensland (2019000700/2018/926),

\section{Strengths and limitations of this study}

- This multicentre pragmatic randomised controlled trial will provide high level evidence of the effectiveness and cost-effectiveness of a novel stratified clinical PAthway of CarE (PACE programme) vs usual care for patients with common musculoskeletal (MSK) pain presentations.

- Development of a custom-designed online evidenceinformed resource will support the implementation of the PACE programme.

- The trial is powered for between-arm subgroup analysis based on risk of poor outcome (low/high).

- Attempts to avoid contamination will be made (eg, no influence over usual care group), but cannot be guaranteed due to the pragmatic nature of this trial.

- Networks and skills developed by clinicians engaged during this trial will enable scaling implementation of PACE for the management for MSK conditions Australia wide.

University of Melbourne (1954239), Curtin University (HRE2019-0263) and Northern Sydney Local Health District (2019/ETH03632). Dissemination of findings will occur via peer-reviewed publications, conference presentations and social media.

Trial registration number ACTRN12619000871145.

\section{INTRODUCTION}

In Australia, musculoskeletal (MSK) conditions including low back pain (LBP), neck pain/whiplash associated disorders (WAD), and knee osteoarthritis (OA) are the highest contributors to years lived with disability, above mental health conditions, respiratory 
and cardiovascular diseases. ${ }^{1}$ Despite this, the leading recommendations to manage these conditions are not followed by most Australians. ${ }^{2}$ The multiple clinical guidelines for the three MSK conditions ${ }^{3-5}$ are poorly implemented in primary care where these conditions are predominantly managed. ${ }^{6}$ General practitioners (GPs) have traditionally been the gatekeeper for access to health services for people with MSK conditions. The overwhelming information that GPs receive is one reason that translation of best practice for patients with MSK conditions is challenging. This is reflected by Australian data where appropriate advice on management of LBP is provided in only $21 \%$ of GP consultations, ${ }^{7}$ and over $50 \%$ of people with neck pain receive unnecessary imaging. ${ }^{8}$ Further, although clinical guidelines for the management of knee OA across the globe universally recommend exercise therapy, ${ }^{5}$ only $13 \%$ of patients with knee OA receive this information. ${ }^{9}$ The impact is a large economic burden due to overuse of medication, ${ }^{10}$ diagnostic imaging ${ }^{7}$ and surgery. ${ }^{11}$

Healthcare costs are higher in patients with more complex MSK presentations, such as those with comorbidities $^{12}$ or coexisting psychological distress, ${ }^{13}$ where recovery from an episode of pain, or flare of symptoms, may be slower. To date, prognostic studies have found that people with $\mathrm{LBP},{ }^{14}$ idiopathic neck pain, ${ }^{15} 16$ $\mathrm{WAD},{ }^{17-20}{\text { knee } \mathrm{OA}^{21} 22}^{22}$ and other MSK pain conditions ${ }^{23}$ who have high levels of pain, disability and psychological distress are more likely to develop persistent pain and disability. This knowledge has led to the development and validation of simple prognostic screening tools to identify people at risk of poor outcome. These include the Keele STarT Back tool for $\mathrm{LBP}^{24}$ the WhipPredict for WAD, ${ }^{25}$ and the Short Form Örebro Musculoskeletal Pain Screening Questionnaire (SF-ÖMSPQ) ${ }^{26}$ and Keele STarT MSK tool ${ }^{27}$ for broader populations of patients with MSK pain.

Stratified care that involves identification of patients' risk of poor outcome and matching treatments based on their prognosis is gaining momentum in MSK healthcare. Essentially, less healthcare is provided to those at low risk of poor outcome, whereas more comprehensive care is provided to those at high risk. Stratified care for LBP has been shown to be clinically effective, cost-effective and implementable in the $\mathrm{UK}^{24} 28$ but adapted models tested in the US have not demonstrated benefits. ${ }^{29}{ }^{30} \mathrm{In}$ Australia, preliminary evidence suggests that a stratified approach improves outcomes for people with MSK pain in a workers compensation setting, ${ }^{23}$ and for people with acute whiplash. ${ }^{31}$ To date, this approach has not been tested for a broader MSK population in Australia in a primary care setting.

Patients with a favourable prognosis should do well with care delivered by a primary healthcare professional (HCP), supported by high quality and credible selfmanagement information resources. ${ }^{24} 283233$ One of the barriers for implementation is the volume of options of internet-based resources, many of which have low credibility standards and contain inaccurate information. ${ }^{34}$ Best practice recommendations are similar for most MSK conditions. These include advice about selfmanagement, remain active and exercise, avoid unnecessary imaging and identify those at risk of persistent pain and disability, and appropriately refer for specialist care. One solution to translate these key messages is to provide access to high quality, evidence-based resources in one location. Such an innovation is lacking in primary care and may improve management for patients with good prognosis.

Conversely, patients at high risk of persistent pain and disability are likely to require more comprehensive assessment, followed by targeted interventions that address multiple and/or complex contributing obstacles to recovery. Allied health clinicians (eg, physiotherapists) with expertise in managing MSK conditions are increasingly used in this secondary referral role. In tertiary care settings, outcomes have included improved efficiency of care and lower cost without compromising patient satisfaction with care. ${ }^{35-39}$ Internationally, physiotherapistdelivered models of care for $\mathrm{OA}$ have demonstrated significant reductions in medication use, sick leave and pain. ${ }^{40-42}$ Bishop et al ${ }^{43}$ demonstrated non-inferior clinical outcomes for people with MSK conditions with physiotherapy-led care compared with usual GP-led care. These, and other studies, ${ }^{445}$ suggest that physiotherapists could reduce pressure on an over-burdened medical system, by providing such services to people with MSK conditions who are at risk of a poor outcome.

In Australian primary healthcare settings, models of care using expert allied health MSK clinicians in this secondary referral role, have not been evaluated in a randomised controlled trial. In a workers' compensation clinical study, management of patients at high risk by allied health clinicians reduced the cost of care by $30 \% .^{2346}$ Further, Australian regulatory bodies ${ }^{47-51}$ support referral of people at high risk of poor outcomes to expert MSK physiotherapists. Physiotherapists who most frequently provide these services are recognised clinical specialists in MSK physiotherapy (MSK specialists), ${ }^{52}$ and findings to date have demonstrated that this role for MSK specialists is feasible and acceptable to primary HCPs and MSK specialists. $^{53}$

This trial, therefore, will combine three novel concepts to provide a solution for the delivery of healthcare services for people with MSK conditions in Australia. First, it will use a prognostic screening tool (SF-ÖMSPQ),${ }^{26}$ validated in Australian cohorts ${ }^{2326}$ to identify those at low and high risk of poor outcome. Second, it will identify and consolidate evidence-based resources in one convenient location to assist management of those at low risk. Finally, it will use the services of MSK specialists in primary care to lead care for patients at high risk. Implementation of this novel stratified PAthway of CarE (hereafter referred to as PACE programme) could potentially improve health outcomes and reduce the burden on the current healthcare system by reducing unnecessary healthcare utilisation and costs. 


\section{Aims}

The primary aim of this trial is to evaluate the effectiveness of the PACE programme on physical health (measured by the physical component subscale (PCS) of the SF12, SF12-PCS) compared with usual care for people with MSK conditions at 12 months. Secondary aims are to evaluate: (1) the effect of the PACE programme vs usual care on other health outcomes (eg, pain self-efficacy, physical disability, general and psychological health) at 3, 6 and 12 months; (2) cost-effectiveness of the care pathway compared with usual care at 12 months and (3) health outcomes between risk subgroups in each arm.

We hypothesise that the PACE programme will result in improved physical health (SF12-PCS) compared with usual care. Secondary hypotheses are that the PACE programme will result in better pain self-efficacy, general and psychological health than usual care at all time points. The PACE programme should deliver treatment more efficiently, determined through our health economic analysis. Finally, we hypothesise that people at high risk in the PACE programme will have improved health outcomes compared with the high-risk matched subgroup in usual care.

\section{METHODS AND ANALYSIS}

\section{Study design and setting}

The study is a multicentre pragmatic randomised controlled trial conducted in primary care, with embedded observational and qualitative research methods to evaluate patients' experiences, HCP practice and collaboration.

\section{Participants}

Participants $(n=716)$ with LBP, neck pain or WAD, or knee OA will be recruited from primary care clinics, hospital emergency departments, and the community in four Australian states (New South Wales, Queensland, Victoria, Western Australia). The number enrolled per condition is anticipated to reflect the population prevalence of each condition.

The process for identification of potential participants will differ depending on the setting. In primary care, HCPs who have indicated interest in assisting with recruitment will provide potential participants with information about the trial. At participating hospitals, potential participants will be identified either directly (eg, in the emergency department or on presentation to an outpatient clinic) or via medical record review. Those who agree to receive further information will be sent details about the trial. Participants will also be recruited from the community via advertisements placed on social media, university bulletins and print media. Those who respond to the invitation will be contacted via telephone, provided with information about the trial, screened for eligibility, and if eligible, invited to participate. Those who agree to participate will be asked to provide informed written consent to participation in the trial (see online supplemental file 1).

\section{Inclusion criteria}

Participants will be eligible for inclusion if they are within 4 weeks of seeking care (or are planning to seek care) for their symptoms and are proficient in written and spoken English. Participants with LBP must fulfil criteria for non-specific $\mathrm{LBP}^{54}$ with/ without associated leg symptoms, and be aged over 18 years. ${ }^{55}$ Participants with neck pain or WAD must fulfil criteria for non-specific neck pain ${ }^{56}$ and/or grade I-III $\mathrm{WAD}^{57}$ and be aged over 18 years. Inclusion criteria for participants with knee OA will be confirmed using the National Institute for Health and Clinical Excellence criteria (at least 45 years old, experience activity-related joint pain and have either no morning joint-related stiffness or morning stiffness lasting $<30 \mathrm{~min}) .^{58}$ Participants at all stages of knee OA who have experienced pain for longer than 3 months will be eligible.

\section{Exclusion criteria}

Exclusion criteria include known or suspected serious medical conditions (eg, cancer, metastatic disease); diagnosed inflammatory (eg, psoriatic arthritis, ankylosing spondylitis) or neurological conditions (eg, Parkinson's disease, stroke); confirmed fracture or dislocation at time of injury (eg, WAD IV); or severe depression defined as at risk of self-harm (answering 'yes' to Item 9 of the Patient Health Questionnaire-9). People with knee OA will be excluded if they have undergone or are scheduled for joint replacement surgery on the knee that is currently painful.

\section{Primary HCPs}

Participants will be asked to nominate the primary HCP who is the main person managing their condition. Primary HCPs could be registered GPs or allied health professionals (eg, physiotherapists, chiropractors, osteopaths). Nominated primary HCPs will be informed of the involvement of their patient in the trial and will be provided with a separate primary HCP information statement explaining the patient's journey in the trial and inviting them to be involved in the embedded HCP observational study.

\section{MSK specialists}

MSK specialists were recruited by the research team prior to trial commencement. Eligible specialist MSK clinicians were defined a priori as HCPs who have a higher degree or fellowship qualifications in MSK health or who have recognised expertise in the management of complex MSK conditions. We anticipated these to mostly be physiotherapists, however, psychologists, chiropractors and medical practitioners were also eligible. Prior to commencement, the MSK specialists underwent a 2-day training workshop developed and delivered by the chief investigators and other experts in the field of MSK pain. The workshop was held in each of the four Australian states involved in the trial. Workshop content and methods used included peer 
Table 1 Questionnaires completed by participants

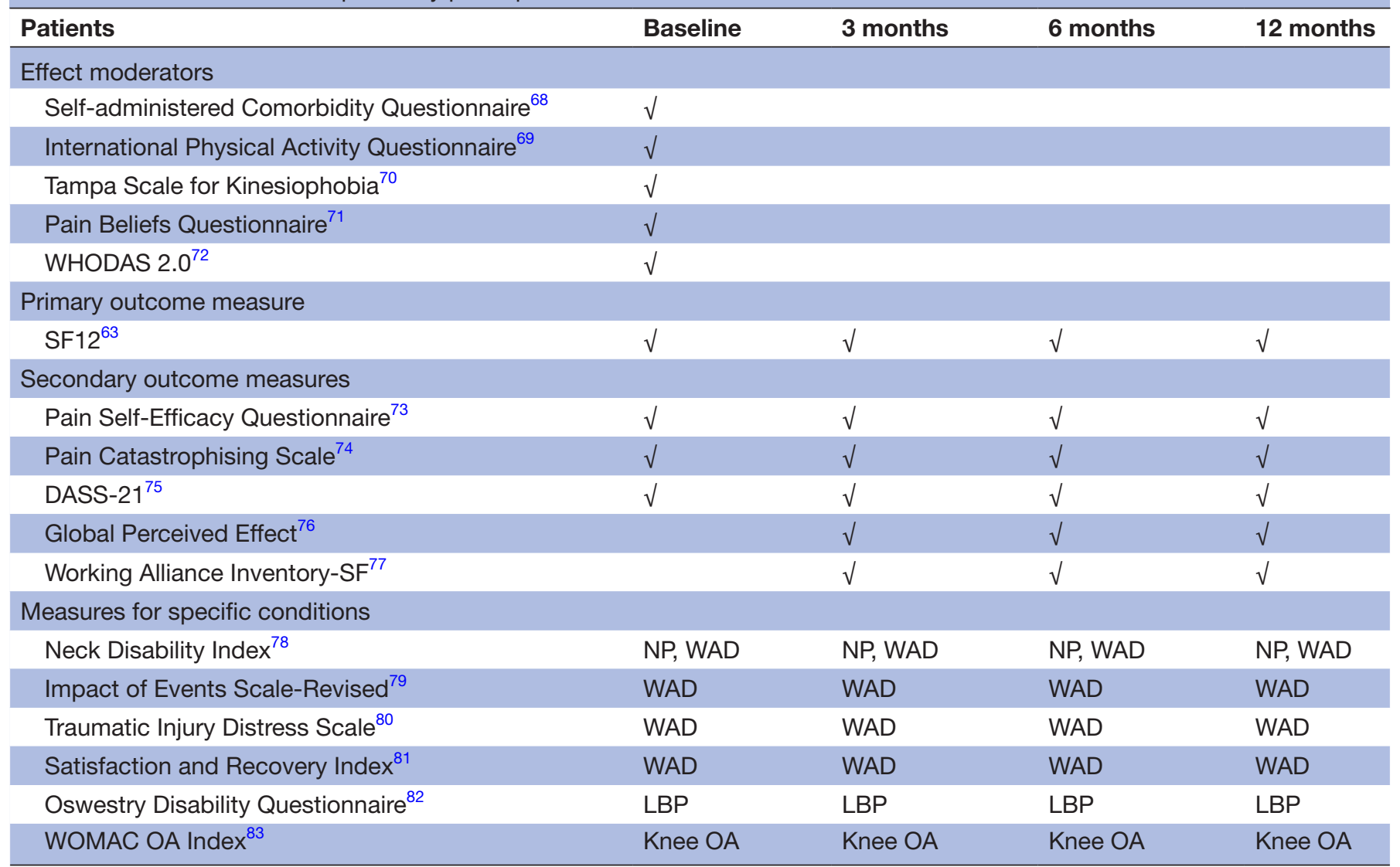

.DASS-21, Depression Anxiety and Stress Scale.; LBP, low back pain; NP, neck pain; OA, osteoarthritis; SF12, 12-Item Short Form; WAD, whiplash associated disorders; WHODAS, WHO Disability Assessment Schedule; WOMAC, Western Ontario and McMaster Universities Osteoarthritis Index.

review of complex cases to identify and address behaviours compliant/non-compliant with the PACE programme.

\section{Procedure}

Participants will complete baseline questionnaires that assess demographic factors, pain and disability, and potential effect moderators. Demographic information will include age, height, weight, occupation, employment status and income and level of education. Participants will be asked to provide contact details of their primary $\mathrm{HCP}(\mathrm{s})$, information about healthcare expenditure (eg, costs of healthcare appointments, imaging etc over the previous 4 weeks) and indirect costs (eg, home help or work absence). Participants will be asked to complete questionnaires relating to pain, disability, health status and known effect moderators (table 1 ).

\section{Stratification and randomisation}

Participants will then complete the SF-ÖMSPQ and be stratified into low risk (scores $<50$ ), or high risk (scores $\geq 50$ ) of a poor outcome. ${ }^{24}$ Risk status and MSK condition (LBP, neck pain, WAD, knee OA), will factor in randomisation to the clinical care pathway or usual care (figure 1). The randomisation process will be managed by the National Health and Medical Research Council
(NHMRC) Clinical Trials Centre at The University of Sydney to achieve balance overall with respect to risk subgroup and MSK condition. ${ }^{59}$ Following randomisation, the research team will contact the participant, explain the next steps, and remind participants that outcome measures will be collected at 3, 6 and 12 months follow-up (table 1).

\section{Intervention}

Clinical PAthway of CarE (PACE programme)

Participants randomised to the PACE programme will be offered care matched to their risk subgroup including access to the custom-designed website developed for the trial ( www.mypainhub.com; MyPainHub). MyPainHub was developed following a translational research framework. Steps were development of content by leading MSK researchers, expert clinicians and patients, engagement and feedback from key stakeholders (eg, consumer organisations (Arthritis Australia), and 50 primary HCPs) and refinement after pilot implementation with 12 patients. The purpose of the website is to provide one location for credible information about MSK disorders suitable for both patients and clinicians. Patient content is structured under risk-based recovery pathways, condition-specific health information (eg, lifestyle, 


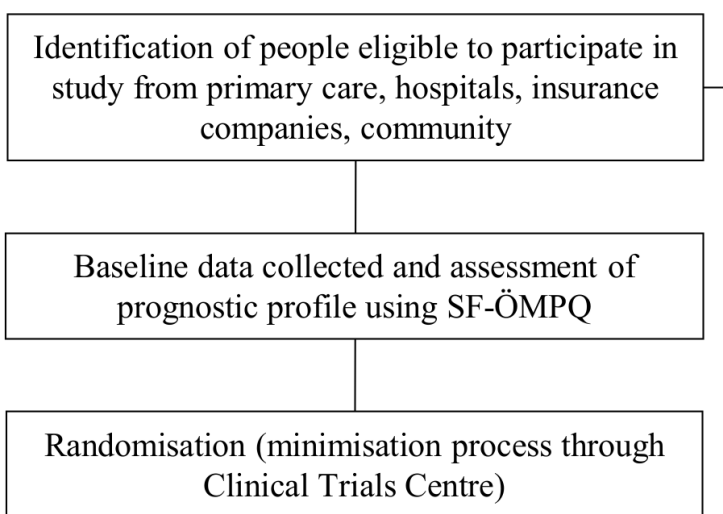
Clinical Trials Centre)

Screen for inclusion criteria:

- Within 4 weeks of seeking care or planning to seek care

- Proficient in written and spoken English Who have:

a. Non-specific low back pain, with or without leg pain, and are over 18 years OR b. Non-specific neck pain $\mathbf{O R}$ whiplash (WAD I-III) over 18 years OR c. Knee OA (NICE criteria), pain longer than 3 months and over 45 years

\section{Exclusion criteria:}

- Known or suspected serious spinal pathology

- Confirmed fracture or dislocation

- Extreme depression (at risk of self-harm)

- Patient with knee OA who have undergone or are scheduled for joint replacement surgery

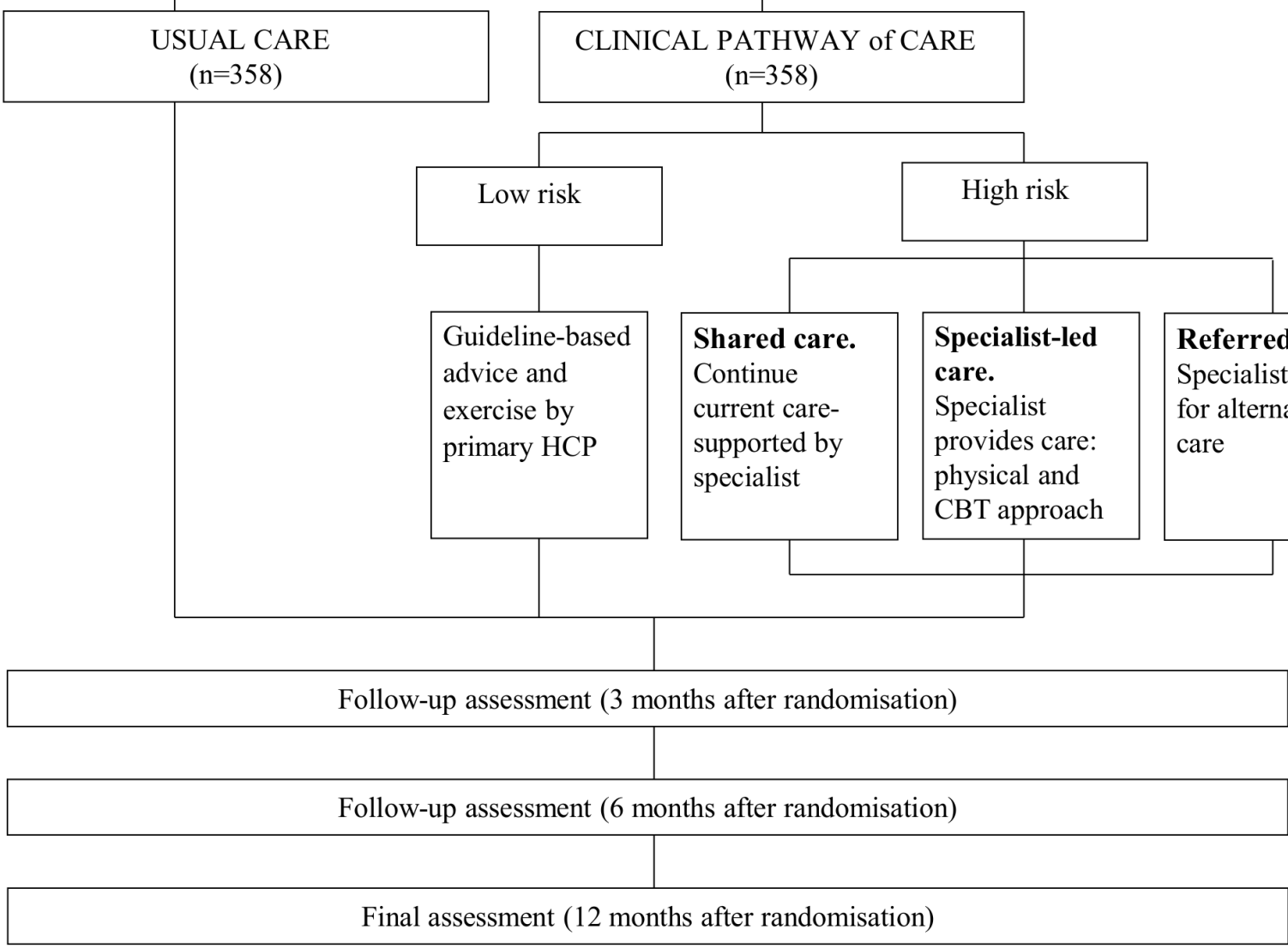

Figure 1 Trial design. HCP, healthcare professional; NICE, National Institute for Health and Clinical Excellence; OA, osteoarthritis; SF-ÖMSPQ, Short Form Örebro Musculoskeletal Pain Screening Questionnaire; WAD, whiplash associated disorders; CBT, cognitive behavioural therapy

sleep), exercises and imaging. Clinician information is structured under assessment, prognosis and risk-based management. Currently, MyPainHub contains 133 links to existing evidence-based resources, 82 references and 20 videos.

\section{Intervention for those at low risk of poor outcome}

Participants at low risk of poor outcome will be directed by our research team to refer to MyPainHub for advice and information about suitable exercises. Their prognosis 
will be explained by telephone initially, with a follow-up call at 6 weeks. The primary HCPs managing these participants will be contacted by the research team by phone or email. Primary HCPs will also be encouraged to use the website and to provide minimal care (up to three sessions of guideline-based care focusing on education, advice to stay active, exercises, developing self-management strategies and discouraging imaging). The number and timing of sessions delivered will be at the discretion of the primary HCP and may depend on factors such as symptoms, specific impairments, and logistics.

\section{Intervention for those at high risk of poor outcome}

Participants stratified as high risk will be offered a referral to a MSK specialist by the research team within 4 weeks of randomisation. Appointments with MSK specialists will be funded by the trial. Participants, their primary HCP and the MSK specialist will all have access to MyPainHub. The MSK specialist will undertake an examination either face-to-face or by telehealth. Telehealth as a mode of specialist health service delivery is an option in Australia, given both the geography of Australia and that this trial commenced during the COVID-19 pandemic. The specialist examination will be tailored to the individual's presentation and will include assessment of factors known to be associated with poor outcome such as; physical impairments, comorbidities, ${ }^{6061}$ pain features, ${ }^{61}$ cognitive and affective factors, ${ }^{31}$ social and lifestyle considerations and participants' values and preferences. ${ }^{62}$ The specialist MSK clinician will discuss the outcome of the assessment with the patient and the primary HCP, develop an individualised care plan and collaboratively decide on one of three further care pathways:

1. Shared care (liaison with primary HCP) is defined as the participant continuing care with their primary HCP, with the MSK specialist monitoring progress. This pathway is used most frequently and is chosen when the team collaboratively agree that the primary HCP has the confidence and expertise to manage the participant's presentation, or it is pragmatic to do so. Example scenarios include when the participant has localised symptoms or moderate scores on screening questionnaires (eg, between 50 and 65 on the SFÖMSPQ), or when geographical distance means primary HCP management is more feasible.

2. Specialist care (short course of specialist-led care) is where the specialist provides a short course (up to six sessions) of care. Care typically involves interventions targeted to identified risk factors, comprising active approaches of exercise within a psychologically informed cognitive behavioural framework. Based on pilot feasibility studies, ${ }^{32} 53$ this pathway was used when the participant's presentation is more complex or when the primary HCP is less confident in management. Example scenarios are when participants have widespread pain sensitivity, dominant psychosocial factors, or when complex differential diagnosis is needed (eg, neck pain, headache, dizziness). Once key goals are achieved (or six sessions have been provided), patients are referred back to the primary HCP with ongoing management plans.

3. Referred care (referral for appropriate additional specialist medical/psychological care, eg, psychologist, neurosurgeon) is chosen when the MSK specialist identifies factors that they consider would be best managed by additional interdisciplinary referral. For example, referral to a psychologist when scores on screening questionnaires (eg, depression or post-traumatic stress) are very high, referral to a medical specialist for worsening pain or referral to a surgeon for worsening radiculopathy. The specialist MSK clinician will liaise (usually via phone call) with the primary HCP, the referred specialist and patient to collaboratively decide on the best options for care.

\section{Usual care}

Participants randomised to usual, non-stratified care, will receive usual care regardless of their risk subgroup, provided by the participant's primary HCP. The primary HCP will be sent a letter informing them of their patient's involvement in the trial, but not their risk status. Decisions regarding assessment, treatment, and referral to other professionals will depend on clinical judgement. No attempt to change usual care will be made and neither the participant nor the primary HCP will have access to the resources contained within MyPainHub. To minimise contamination between the intervention and usual care arms, primary HCPs will be limited to managing a maximum of three participants in the trial. Data on the usual care provided/received prior to and during the trial will be collected and described.

\section{Outcome measures}

Outcomes will be collected at baseline and at 3, 6 and 12 months after randomisation (figure 1). Data will be collected online (or by telephone interview or return post if a participant is unable to access a computer or mobile device). Outcome data will be sought from all randomised participants, regardless of adherence to the trial protocol.

\section{Primary outcome}

The primary health outcome will be physical health (SF12-PCS) ${ }^{63}$ at 12 months.

\section{Secondary outcomes}

Secondary health outcomes will be pain self-efficacy, physical disability, general and psychological health at 3, 6 and 12 months (table 1). Cost-effectiveness is the key health economic outcome of interest. This will be determined by estimating the incremental cos per quality-adjusted life-years gained, calculated using utility weights generated from participants' SF6D responses using the UK weights ${ }^{64}$ or Australian weights if available at trial conclusion. Direct and indirect costs will be calculated. Direct costs (eg, primary HCP visits, pharmaceutical, specialist and imaging services) will be calculated using rebates taken from the Medicare Benefits Schedule, workers 
Table 2 Secondary outcome measures relating to participants' direct and indirect healthcare costs

\begin{tabular}{lllll}
\hline & Baseline & $\mathbf{3}$ months & $\mathbf{6}$ months & $\mathbf{1 2}$ months \\
\hline Imaging & $\sqrt{ }$ & $\sqrt{ }$ & $\sqrt{ }$ & $\sqrt{ }$ \\
Medication & $\sqrt{ }$ & $\sqrt{ }$ & $\sqrt{ }$ & $\sqrt{ }$ \\
$\begin{array}{l}\text { Treatment/ } \\
\text { interventions }\end{array}$ & $\sqrt{ }$ & $\sqrt{ }$ & $\sqrt{ }$ & $\sqrt{ }$ \\
$\begin{array}{l}\text { Travel costs } \\
\begin{array}{l}\text { associated with } \\
\text { treatment }\end{array}\end{array}$ & $\sqrt{ }$ & $\sqrt{ }$ & $\sqrt{ }$ \\
\end{tabular}

$\begin{array}{llll}\begin{array}{l}\text { Home } \\ \text { assistance }\end{array} & \sqrt{ } & \sqrt{ } & \sqrt{ } \\ \text { Time off work/ } & \sqrt{ } & \sqrt{ } & \sqrt{ }\end{array}$

unable to

perform usual

activities

\begin{tabular}{llll}
$\begin{array}{l}\text { Associated } \\
\text { healthcare } \\
\text { expenses }\end{array}$ & $\sqrt{ }$ & $\sqrt{ }$ & $\sqrt{ }$ \\
\hline
\end{tabular}

compensation schemes and the Pharmaceutical Benefits Scheme. Direct healthcare costs (eg, consumer copayments) and non-healthcare costs (eg, work absenteeism), which are not captured by these insurer payments, will be identified using patient cost diaries (table 2).

Outcomes assessed by embedded qualitative and observational studies will include patient experience, clinical care provided and communication and collaboration between primary and specialist HCPs and participants.

\section{Commencement}

Data collection commenced in March 2020 (one participant enrolled) but was suspended due to COVID-19. Data collection recommenced in July 2020 and is scheduled for completion by December 2022.

\section{Patient and public involvement}

Consumers, primary HCPs and specialist MSK clinicians were involved in the assessment of the burden of the time required to complete the baseline questionnaires and outcome measures. Patients, primary HCPs, specialist MSK clinicians and key stakeholders (eg, the Australian Physiotherapy Association, Chiropractic Australia and Arthritis Australia) were engaged to review and provide feedback on MyPainHub. Modifications to the website were made in response to this feedback. Participants will be involved in the embedded qualitative studies and discussion of findings. Participants who consent to be contacted about the results of the trial will be provided with a one-page summary of the findings.

\section{Statistical methods}

The sample size calculation was initially based on the SF12-PCS and the most conservative estimate of the clinically important difference in physical health for the included MSK conditions (LBP; mean change 3.18, SD 7.7).$^{6465}$ Originally, the sample size was inflated fourfold to allow for subgroup analysis by condition (LBP, neck pain/WAD, knee OA). Because of the impact of COVID-19 on recruitment and the trial budget, we recalculated the sample size using the actual SD from the baseline data of the first 220 participants $(\mathrm{SD}=8.5)$. This revealed that a sample size of 304 ( $n=152$ usual care, $n=152$ PACE programme) will achieve $90 \%$ power to reject the null hypothesis of equal means, if the population mean difference between the two trial arms is 3.18 (SD 8.5); significance level (alpha 5\%), effect size overall between trial arms of 0.37 using a two-sided two-sample equal-variance t-test. For sufficient power to conduct sub-group analysis of the high risk subgroup (high risk PACE programme vs high risk usual care, assuming $\sim 40 \%$ of participants are at high risk), a total sample size of 716 is required. This assumes superiority only in the high-risk group, and allows for a $15 \%$ lost to follow-up, hence 179 participants in each subgroup are required (ie, 179 in each of the low and high risk subgroups, in the PACE programme and usual care).

\section{Statistical analysis}

The trial biostatistician (DB) will analyse health outcome data in a blinded manner, and a health economist (LC) will lead the cost-effectiveness analysis. All analyses will be conducted on an intention-to-treat basis without imputation for missing data. However, if there are missing data and the proportion of missing values is less than $20 \%$, the last observation will be carried forward (LOCF). The primary and secondary outcomes will be analysed independently at 3, 6 and 12 months, using generalised linear models, with an appropriate link function, to test for an intervention effect adjusted for the baseline values. The subgroup analyses will be sensitivity analyses. A detailed statistical analysis plan will be available prior to the start of data analysis.

\section{Registration and dissemination}

Trial conduct

The trial will be conducted in full conformance with principles of the Declaration of Helsinki, Good Clinical Practice and within the privacy laws and regulations of Australia. Any modifications to the protocol that may affect the conduct of the trial, potential benefit to the participant or participant safety, including changes to objectives, trial design, patient population, sample size and procedures, will require a formal amendment to the protocol. Such amendment will be agreed on by the research team and approved by the hospital and university ethics committees.

\section{Data storage, access and dissemination and monitoring}

Data will be collected through the University's Research Electronic Data Capture (REDCap) system and stored on secure university Server. Patient identification details used to track participants throughout the trial will be stored in a separate, password-protected file 
on each Universities' research data store accessible by the researchers from the specific university. Data are stored for 20 years as per the Australian Health Data requirements.

Data custodian will be CI Evans, the project manager. Data will be monitored by the research assistant in each state responsible for data collection. As data are collected online and directly entered by participants, the research assistants will check for data quality and missing data on a monthly basis in order to ensure all available data are entered or captured. The final data set will be managed and cleaned by CI Evans. It will be deidentified then sent to the trial biostatistician (CI Black) for analysis. This process is independent from the funding body (NHMRC). The final trial dataset will be accessible by The University of Sydney, with access provided to the CI's at the other participating institutions being: The University of Queensland, University of Melbourne, Curtin University.

\section{Trial governance and monitoring}

The trial will be overseen by a steering committee comprising CIA Rebbeck and the CI responsible for each site (QLD CI Sterling, VIC CI Bennell, WA CI Beales NSW CI Simic). Coordination centre will be The University of Sydney, managed by CI Rebbeck, CI Evans (project manager), CI Simic and CI Ferreira. The coordination centre will be responsible for the lead ethics application, setting up the database in REDCap, writing the protocol, coordinating the randomisation process, training trial staff and training specialist MSK clinicians to deliver the intervention. The site leaders will be responsible for managing the trial in their state including recruitment and management of staff within their state, ethics at their institution, site specific ethics at participating hospitals, participant recruitment and follow-up within their state and coordination of the intervention. The steering committee will meet fortnightly to discuss the trial, progress and monitoring. The project manager will additionally liaise with project staff on a weekly basis to follow-up on specific actions. Site leads may conduct site-specific meetings as required.

The steering committee will report twice yearly to the whole project team comprising all CIs. Any issues unable to be resolved by the steering committee will be brought to the project team, adjudicated by CI Refshauge.

\section{Adverse event management}

We will follow the safety in clinical trial guidelines as outlined by The University of Sydney guidelines. This will include reporting any adverse event to HREC and the Clinical Trials Governance (CTG) Team within 72 hours of the event occurring, using the Safety Report Form for clinical trials.

Anticipated adverse events would be a minor exacerbation of symptoms with the examinations undertaken by our clinical specialists and/or mild psychological distress for completing our questionnaires. All interventions in this trial will be administered by registered practising HCPs in primary care. The primary HCP will be able to manage the adverse event in the first instance. They will either be the participant's own primary HCP, hence will manage as per best practise, or they will be a specialist treating the patient then liaising with the primary HCP. All clinicians in our trial will understand to report the adverse event to the site CI and the site CI will follow the safety report guidelines by their administering institution.

\section{Trial audit}

The conduct and auditing of this trial will be overseen by the CTG at the University of Sydney, a team independent of the investigators and the NHMRC (trial sponsor).

\section{DISCUSSION}

This paper details the protocol for a multi-centre pragmatic randomised controlled trial that will compare the outcomes from a novel stratified clinical (PACE programme) with usual care. The novelty of the PACE programme is the combination of three concepts that aim to deliver health services more efficiently. First is the use of the SF-ÖMPSQ to stratify people with common MSK conditions to low and high risk of poor outcome. Second is the provision of minimal care to those at low risk supported by credible and accessible evidence-based resources. Third is to use MSK specialists to coordinate and deliver care for patients at high risk, selecting one of three matched interventions (shared, specialist referred care) between the HCP, MSK specialists and medical/ psychology specialist clinicians.

Evidence supports the notion that people with MSK pain who are at low risk of poor outcome are likely to do well with guideline-based information and simple exercises delivered by primary HCPs. ${ }^{27} 65$ However, those at high risk of poor outcome may benefit from more comprehensive care from clinicians with expertise in managing MSK conditions who could then help the patient and primary HCP decide on the best course of care.$^{66}$ Based on the improved health service delivery demonstrated with MSK specialist models of care in tertiary settings, 363967 we hypothesise that the same efficiency of care will occur by implementation of the PACE programme in primary care. Specifically, to avoid over-use of healthcare for those at low risk, and to provide earlier access to more appropriate and more intensive care for those at high risk.

The trial has already resulted in the development of MyPainHub to support participants and their HCPs in the implementation of the clinical care pathway. This resource will become publicly available after trial completion. Stakeholder consultation has led to establishment and consolidation of key relationships between industry, clinicians, researchers and professional bodies. Outcomes from this trial will establish a strong and collaborative network of 
clinicians capable of scaling the PACE programme across Australia at the trial completion.

\section{Author affiliations}

${ }^{1}$ School of Health Sciences, Faculty of Medicine and Health, The University of Sydney, Camperdown, New South Wales, Australia

2John Walsh Centre for Rehabilitation Research, New South Wales, Australia, Kolling Institute, Northern Sydney Local Health District and Faculty of Medicine and Health, The University of Sydney, St Leonards, New South Wales, Australia

${ }^{3}$ Healthia Limited, Brisbane, Queensland, Australia

${ }^{4}$ School of Physiotherapy and Exercise Science, Curtin University, Perth, Western Australia, Australia

${ }^{5}$ RECOVER Injury Research Centre, The University of Queensland, Brisbane, Queensland, Australia

${ }^{6}$ NHMRC Centre of Research Excellence in Road Traffic Injury Recovery, The University of Queensland, Brisbane, Queensland, The University of Queensland, Brisbane, Queensland, Australia

${ }^{7}$ Centre for Health, Exercise and Sports Medicine, The University of Melbourne, Melbourne, Victoria, Australia

${ }^{8}$ Pain Management Research Institute, Kolling Institute, Northern Sydney Local Health District and Faculty of Medicine and Health, The University of Sydney, St Leonards, New South Wales, Australia

${ }^{9}$ Faculty of Health and Behavioural Sciences, The University of Queensland, Brisbane, Queensland, Australia

${ }^{10}$ Department of Sociology and Business Law, The University of Queensland, Centre for the Business and Economics of Health, The University of Bologna, Bologna, Emilia-Romagna, Italy

${ }^{11}$ Primary Care Centre Versus Arthritis, School of Medicine, Keele University, Keele, UK

${ }^{12}$ Surgical, Treatment and Rehabilitation Service (STARS) Education and Research Alliance, The University of Queensland and Metro North Hospital and Health Service, Brisbane, Queensland, Australia

Correction notice This article has been corrected since it was first published. The affiliation section has been updated.

Twitter Trudy Rebbeck @TrudyRebbeck, Darren Beales @DBealesPhysio, Julia Treleaven @JuliaTreleaven, Lyndal Trevena @LyndalTrevena and Deborah Black @ debannblack

Contributors Conceptualisation: TR, PF, MSt, KLB, IC, MN, CR, GJ, JT, LT, KR, LC, NF, PH, MF, TJS and MSi. Funding acquisition: TR, PF, MSt, KLB, IC, MN, CR, GJ, JT, LT, KR, LC, NF, PH, MF, TJS and MSi. Development of protocol: TR, MSi, DB, KE. Writing - original draft: TR and KE. Writing-review and editing: The final version of the protocol was approved by all the authors.

Funding This work is supported by The National Health and Medical Research Council (NHMRC, APP1141377). KLB (APP1174431) and PH (APP1194937) are supported by NHMRC Investigator grants. MSi is supported by the Sydney Research Accelerator Fellowship. TR, PF and MF are supported by NHMRC Career Development Fellowships. MSt receives unrestricted grant funding from the Motor Accident Insurance Commission of Queensland.

Disclaimer The funding source has no role in trial design, data collection, analysis and interpretation, in the writing of the report or in the decision to submit the paper for publication.

Competing interests $\mathrm{DB}$ is director of a clinic providing specialist physiotherapy services in primary care. At the time of this trial, DB was the President of the Australian College of Physiotherapists, the organisation responsible for the training of specialist physiotherapists in Australia.

Patient consent for publication Not applicable.

Provenance and peer review Not commissioned; peer reviewed for ethical and funding approval prior to submission.

Supplemental material This content has been supplied by the author(s). It has not been vetted by BMJ Publishing Group Limited (BMJ) and may not have been peer-reviewed. Any opinions or recommendations discussed are solely those of the author(s) and are not endorsed by BMJ. BMJ disclaims all liability and responsibility arising from any reliance placed on the content. Where the content includes any translated material, BMJ does not warrant the accuracy and reliability of the translations (including but not limited to local regulations, clinical guidelines, terminology, drug names and drug dosages), and is not responsible for any error and/or omissions arising from translation and adaptation or otherwise.

Open access This is an open access article distributed in accordance with the Creative Commons Attribution Non Commercial (CC BY-NC 4.0) license, which permits others to distribute, remix, adapt, build upon this work non-commercially, and license their derivative works on different terms, provided the original work is properly cited, appropriate credit is given, any changes made indicated, and the use is non-commercial. See: http://creativecommons.org/licenses/by-nc/4.0/.

\section{ORCID iDs}

Trudy Rebbeck http://orcid.org/0000-0002-4453-4739

Kerrie Evans http://orcid.org/0000-0002-7592-7850

Darren Beales http://orcid.org/0000-0002-7176-4644

Carrie Ritchie http://orcid.org/0000-0002-7072-0148

Lyndal Trevena http://orcid.org/0000-0003-1419-1832

Deborah Black http://orcid.org/0000-0001-9174-1565

\section{REFERENCES}

1 Hoy WE. Australian burden of disease study: impact and causes of illness and death in Australia 2011. Australian burden of disease study series. Canberra: Australian Instittue of Health and Welfare, 2016.

2 Pink B, Allbon P. Health and welfare of Australia's Aboriginal and Torres Strait Islander peoples. 3. Canberra: Australian Institute of Health and Welfare and Australian Bureau of Statistics, 2008.

3 State Insurance Regulatory Authority. Guidelines for the management of acute whiplash-associated conditions for health professionals. 3 edn. Sydney, 2014

4 Koes BW, van Tulder M, Lin C-WC, et al. An updated overview of clinical guidelines for the management of non-specific low back pain in primary care. Eur Spine J 2010;19:2075-94.

5 Nelson AE, Allen KD, Golightly YM, et al. A systematic review of recommendations and guidelines for the management of osteoarthritis: the chronic osteoarthritis management initiative of the U.S. bone and joint initiative. Semin Arthritis Rheum 2014;43:701-12.

6 Lin I, Wiles LK, Waller R, et al. Poor overall quality of clinical practice guidelines for musculoskeletal pain: a systematic review. Br J Sports Med 2018;52:337-43.

7 Williams CM, Maher CG, Hancock MJ, et al. Low back pain and best practice care: a survey of general practice physicians. Arch Intern Med 2010;170:271-7.

8 Bandong AN, Leaver A, Mackey M, et al. Adoption and use of guidelines for whiplash: an audit of insurer and health professional practice in New South Wales, Australia. BMC Health Serv Res 2018;18:622.

9 Jordan KM, Sawyer S, Coakley P, et al. The use of conventional and complementary treatments for knee osteoarthritis in the community. Rheumatology 2004;43:381-4.

10 Kingsbury SR, Hensor EMA, Walsh CAE, et al. How do people with knee osteoarthritis use osteoarthritis pain medications and does this change over time? Data from the osteoarthritis initiative. Arthritis Res Ther 2013;15:R106.

11 Laupattarakasem W, Laopaiboon M, Laupattarakasem P, et al. Arthroscopic debridement for knee osteoarthritis. Cochrane Database Syst Rev 2008:CD005118.

12 Lowe DB, Taylor MJ, Hill SJ. Associations between multimorbidity and additional burden for working-age adults with specific forms of musculoskeletal conditions: a cross-sectional study. BMC Musculoskelet Disord 2017;18:135.

13 Pinheiro MB, Ferreira ML, Refshauge K, et al. Symptoms of depression and risk of new episodes of low back pain: a systematic review and meta-analysis. Arthritis Care Res 2015;67:1591-603.

14 Rabey M, Smith A, Beales D, et al. Multidimensional prognostic modelling in people with chronic axial low back pain. Clin J Pain 2017;33:877-91.

15 Hush JM, Lin CC, Michaleff ZA, et al. Prognosis of acute idiopathic neck pain is poor: a systematic review and meta-analysis. Arch Phys Med Rehabil 2011;92:824-9.

16 Leaver AM, Maher CG, McAuley JH, et al. People seeking treatment for a new episode of neck pain typically have rapid improvement in symptoms: an observational study. J Physiother 2013;59:31-7.

17 Rebbeck T, Sindhusake D, Cameron ID, et al. A prospective cohort study of health outcomes following whiplash associated disorders in an Australian population. Inj Prev 2006;12:93-8. 
18 Kamper SJ, Rebbeck TJ, Maher CG, et al. Course and prognostic factors of whiplash: a systematic review and meta-analysis. Pain 2008;138:617-29.

19 Sterling M. Developmental trajectories of pain/disability and PTSD symptoms after whiplash injury. Pain 2010;150:22-8.

20 Sterling M, Hendrikz J, Kenardy J, et al. Assessment and validation of prognostic models for poor functional recovery 12 months after whiplash injury: a multicentre inception cohort study. Pain 2012:153:1727-34

21 de Rooij M, van der Leeden M, Heymans MW, et al. Prognosis of pain and physical functioning in patients with knee osteoarthritis: a systematic review and meta-analysis. Arthritis Care Res 2016;68:481-92.

22 Fransen M, Simic M, Harmer AR. Determinants of MSK health and disability: lifestyle determinants of symptomatic osteoarthritis. Best Pract Res Clin Rheumatol 2014;28:435-60.

23 Nicholas MK, Costa DSJ, Linton SJ, et al. Implementation of Early Intervention Protocol in Australia for 'High Risk' Injured Workers is Associated with Fewer Lost Work Days Over 2 Years Than Usual (Stepped) Care. J Occup Rehabil 2020;30:93-104.

24 Hill JC, Whitehurst DGT, Lewis M, et al. Comparison of stratified primary care management for low back pain with current best practice (start back): a randomised controlled trial. Lancet 2011;378:1560-71.

25 Ritchie C, Hendrikz J, Jull G, et al. External validation of a clinical prediction rule to predict full recovery and ongoing moderate/severe disability following acute whiplash injury. J Orthop Sports Phys Ther 2015;45:242-50.

26 Linton SJ, Nicholas M, MacDonald S. Development of a short form of the Örebro musculoskeletal pain screening questionnaire. Spine 2011;36:1891-5.

27 Nguyen $\mathrm{H}$, Rebbeck T, Kifley A, et al. Positive recovery for low-risk injuries screened by the short form - Örebro musculoskeletal pain screening questionnaire following road traffic injury: evidence from an inception cohort study in New South Wales, Australia. BMC Musculoskelet Disord 2019;20:531.

28 Foster NE, Mullis R, Hill JC, et al. Effect of stratified care for low back pain in family practice (impact back): a prospective population-based sequential comparison. Ann Fam Med 2014;12:102-11.

29 Cherkin D, Balderson B, Wellman R, et al. Effect of low back pain risk-stratification strategy on patient outcomes and care processes: the match randomized trial in primary care. $J$ Gen Intern Med 2018;33:1324-36.

30 Delitto A, Patterson CG, Stevans JM, et al. Stratified care to prevent chronic low back pain in high-risk patients: the target trial. A multi-site pragmatic cluster randomized trial. EClinicalMedicine 2021;34:100795.

31 Sterling M, Smeets R, Keijzers G, et al. Physiotherapist-delivered stress inoculation training integrated with exercise versus physiotherapy exercise alone for acute whiplash-associated disorder (StressModex): a randomised controlled trial of a combined psychological/physical intervention. Br J Sports Med 2019;53:1240-7.

32 Bandong AN, Mackey M, Leaver A, et al. An interactive website for whiplash management (my whiplash navigator): process evaluation of design and implementation. JMIR Form Res 2019;3:e12216.

33 Hodges PW, Setchell J, Nielsen M. An Internet-based consumer resource for people with low back pain (MyBackPain): development and evaluation. JMIR Rehabil Assist Technol 2020;7:e16101.

34 Ferreira G, Traeger AC, Machado G, et al. Credibility, accuracy, and comprehensiveness of Internet-based information about low back pain: a systematic review. J Med Internet Res 2019;21:e13357.

35 Blackburn MS, Cowan SM, Cary B, et al. Physiotherapy-led triage clinic for low back pain. Aust Health Rev 2009;33:663-70.

36 Desmeules F, Roy J-S, MacDermid JC, et al. Advanced practice physiotherapy in patients with musculoskeletal disorders: a systematic review. BMC Musculoskelet Disord 2012;13:107.

37 Hart DL, Dobrzykowski EA. Influence of orthopaedic clinical specialist certification on clinical outcomes. J Orthop Sports Phys Ther 2000;30:183-93.

38 Oldmeadow LB, Bedi HS, Burch HT, et al. Experienced physiotherapists as gatekeepers to hospital orthopaedic outpatient care. Med J Aust 2007;186:625-8.

39 Rymaszewski LA, Sharma S, McGill PE, et al. A team approach to musculo-skeletal disorders. Ann R Coll Surg Engl 2005;87:174-80.

40 Skou ST, Odgaard A, Rasmussen JO, et al. Group education and exercise is feasible in knee and hip osteoarthritis. Dan Med $J$ 2012;59:A4554.

41 Roos EM, Skou ST, Grønne DT. GLA:D®. Annual Report 2017, 2017.
42 Tan SS, Teirlinck CH, Dekker J, et al. Cost-utility of exercise therapy in patients with hip osteoarthritis in primary care. Osteoarthritis Cartilage 2016;24:581-8.

43 Bishop A, Chen Y, Protheroe J, et al. Providing patients with direct access to musculoskeletal physiotherapy: the impact on general practice musculoskeletal workload and resource use. The STEMS-2 study. Physiotherapy 2021;111:48.

44 NHS trust development authority: annual report and accounts 2016/2017, UK

45 O'Cathain A, Froggett M, Taylor MP. General practice based physiotherapy: its use and effect on referrals to hospital orthopaedics and rheumatology outpatient departments. Br J Gen Pract 1995;45:352-4.

46 Beales D, O'Sullivan P. Beliefs of Australian physical therapists related to lumbopelvic pain following a biopsychosocial workshop. $J$ Phys Ther Educ 2014;28:128-33.

47 Motor Accidents Authority. Guidelines for the management of whiplash associated conditions for health professionals. 2nd ed. Sydney: State Insurance Regulatory Authority, 2007. https://www. sira.nsw.gov.au/

48 State Insurance Regulatory Authority NSW. Motor accidents dispute resolution services, 2016. Available: www.sira.nsw.gov.au

49 Motor Accident Insurance Commission of Queensland (MAIC). Guidelines for compulsory third party rehabilitation providers. Available: www.maic.qld.gov.au

50 Insurance Commission of Western Australia. A guide for people injured in a motor vehicle crash. Available: www.icwa.wa.gov.au

51 Transport Accident Commission of Victoria. Allied health independent medical examiners. Available: www.tac.vic.gov.au

52 Australian College of Physiotherapists. Two-year training program in the process of specialisation in physiotherapy, 2017. Available: https://australian.physio/pd/australian-college-physiotherapyinformation

53 Bandong AN, Leaver A, Mackey M, et al. Referral to specialist physiotherapists in the management of whiplash associated disorders: perspectives of healthcare practitioners. Musculoskelet Sci Prac 2018;34:14-26.

54 Maher C, Underwood M, Buchbinder R. Non-specific low back pain. Lancet 2017;389:736-47.

55 Oliveira CB, Maher CG, Pinto RZ, et al. Clinical practice guidelines for the management of non-specific low back pain in primary care: an updated overview. Eur Spine J 2018;27:2791-803.

56 World Health Organization. International classification of diseases, injuries, and causes of death. 11th edn. Geneva, CH, 2018.

57 Spitzer WO, Skovron ML, Salmi LR, et al. Scientific monograph of the Quebec Task Force on Whiplash-Associated Disorders: redefining "whiplash" and its management. Spine 1995;20:1S-73.

58 National Institute for Health and Clinical Excellence. NICE guideline on osteoarthritis: the care and management of osteoarthritis in adults, NICE clinical guideline 177, 2014. Available: http://guidance. nice.org.uk/CG177

59 Altman DG, Bland JM. Treatment allocation by minimisation. BMJ 2005;330:843.

60 Henschke N, Maher CG, Refshauge KM, et al. Prognosis in patients with recent onset low back pain in Australian primary care: inception cohort study. BMJ 2008;337:a171.

61 Sterling M, Hendrikz J, Kenardy J. Similar factors predict disability and posttraumatic stress disorder trajectories after whiplash injury. Pain 2011:152:1272-8

62 Mitchell T, Beales D, Slater H. Musculoskeletal clinical translation framework: from knowing to doing (e-book). Perth: Curtin University, 2017.

63 Busija L, Pausenberger E, Haines TP. Adult measures of general health and health-related quality of life: medical outcomes study short form 36-Item (SF-36) and short form 12-Item (SF-12) health surveys, Nottingham health profile (NHP), sickness impact profile (SIP), medical outcomes study short form 6D (SF-6D), health Utilities index mark 3 (HUI3), quality of well-being scale (QWB), and assessment of quality of life (AQoL). Arthritis Care Res 2011:63:S383-412.

64 Brazier JE, Roberts J. The estimation of a preference-based measure of health from the SF-12. Med Care 2004;42:10.1097/01.mlr. 0000135827.18610.0d:851-9.

65 Foster NE, Anema JR, Cherkin D, et al. Prevention and treatment of low back pain: evidence, challenges, and promising directions. Lancet 2018;391:2368-83.

66 Rebbeck T, Leaver A, Bandong AN, et al. Implementation of a guideline-based clinical pathway of care to improve health outcomes following whiplash injury (whiplash impact): protocol of a randomised, controlled trial. J Physiother 2016;62:10.1016/j. jphys.2016.02.006 
67 Murphy S, Blake C, Power CK, et al. The role of clinical specialist physiotherapists in the management of low back pain in a spinal triage clinic. Ir J Med Sci 2013;182:10.1007/s11845-0130945-7:643-50.

68 Sangha O, Stucki G, Liang MH, et al. The self-administered comorbidity questionnaire: a new method to assess comorbidity for clinical and health services research. Arthritis Rheum 2003;49:10.1002/art.10993:156-63.

69 Craig CL, Marshall AL, Sjöström M, et al. International physical activity questionnaire: 12 -country reliability and validity. Med Sci Sports Exerc 2003;35:1381-95.

70 Woby SR, Roach NK, Urmston M, et al. Psychometric properties of the TSK-11: a shortened version of the Tampa scale for Kinesiophobia. Pain 2005;117:137-44.

71 Edwards LC, Pearce SA, Turner-Stokes L, et al. The pain beliefs questionnaire: an investigation of beliefs in the causes and consequences of pain. Pain 1992;51:267-72.

72 Rehm J, Üstün TB, Saxena S, et al. On the development and psychometric testing of the WHO screening instrument to assess disablement in the general population. Int J Methods Psychiatr Res 1999;8:110-22.

73 Nicholas MK. The pain self-efficacy questionnaire: taking pain into account. Eur J Pain 2007;11:153-63.

74 Sullivan MJL, Bishop SR, Pivik J. The pain catastrophizing scale: development and validation. Psychol Assess 1995;7:524-32.

75 Lovibond PF, Lovibond SH. The structure of negative emotional states: comparison of the depression anxiety stress scales (DASS) with the Beck depression and anxiety inventories. Behav Res Ther 1995;33:335-43.

76 Dworkin RH, Turk DC, Farrar JT, et al. Core outcome measures for chronic pain clinical trials: IMMPACT recommendations. Pain 2005;113:9-19.

77 Horvath AO, Greenberg LS. Development and validation of the working alliance inventory. J Couns Psychol 1989;36:223-33.

78 Vernon $\mathrm{H}$, Mior S. The neck disability index: a study of reliability and validity. $J$ Manipulative Physiol Ther 1991:14:1834753:409-15.

79 Weiss DS. The Impact of Event Scale: revised. In: Cross-Cultural assessment of psychological trauma and PTSD. Boston, MA: Springer US, 2007: 219-38.

80 Walton DM, Krebs D, Moulden D, et al. The traumatic injuries distress scale: a new tool that quantifies distress and has predictive validity with patient-reported outcomes. J Orthop Sports Phys Ther 2016;46:10.2519/jospt.2016.6594:920-8.

81 Walton DM, MacDermid JC, Pulickal M, et al. Development and initial validation of the satisfaction and recovery index (Sri) for measurement of recovery from musculoskeletal trauma. Open Orthop J 2014;8:316.

82 Fairbank JC, Pynsent PB. The Oswestry disability index. Spine 2000;25:2940-53.

83 Bellamy N. The WOMAC knee and hip osteoarthritis indices: development, validation, globalization and influence on the development of the AUSCAN hand osteoarthritis indices. Clin Exp Rheumatol 2005;23:S148. 